\title{
NETWORK RECONFIGURATION AND CAPACITOR PLACEMENT IN DISTRIBUTION SYSTEM WITH HARMONIC SEARCH ALGORITHM
}

\author{
Pushpendra Singh ${ }^{1}$ and Komal Arora ${ }^{1}$ \\ ${ }^{1}$ Department of Electrical \& Electronics Engineering, Govt.Women Engineering College, \\ Ajmer, Rajasthan,India
}

\begin{abstract}
This paper presents a recently developed approach Harmonic Search Algorithm for optimal reconfiguration and capacitor placement which is successfully applied to optimize radial distribution system with objective of reduction in the power loss for dynamic load. Simulation is carried out on Standard IEEE 33 bus distribution system. Results are compared with popular methods available in the literature like Genetic Algorithm and Ant Colony Optimization. Results show that the proposed algorithm can converge to optimum solution quickly with better accuracy when compare to Genetic Algorithm and Ant Colony Optimization.
\end{abstract}

\section{KEYWORDS}

Distribution System, Network Reconfiguration, Optimal Capacitor Placement, Power Loss Reduction, Radial Network, Heuristic Technique

\section{INTRODUCTION}

A power distribution system is basically a group of radial feeders connected together by various tie line switches and tie lines. Power loss in the power distribution system is a major concern of electric power distribution utilities. Among conventional methods of reducing power losses, optimal reconfiguration and capacitor placement are two most effective methods which can be applied on the distribution system.

Network reconfiguration is the method of changing the topological structure of feeders by altering opening/closing of tie line switching and sectionalizing. Distribution system networks are normally reconfigured to minimize real power loss and to mitigate overload burden in the network. Due to this over loading and real power losses the voltage profile of system are reduces, which should be improved. Capacitors are integrated in distribution network to meet the required level of load demand and improve the voltage profile as well as provide reliable and uninterrupted power supply resulting minimum power loss and economical benefits. Network reconfiguration and capacitor placement in distribution networks are normally considered independently. However, in the proposed method, network reconfiguration and capacitor placement are deal simultaneously for improving loss minimization and voltage profile for dynamic load. Distribution system reconfiguration for loss reduction was first proposed by Merlin and Back [1]. They employed a blend of optimization and heuristics to determine the minimal-loss operating configuration for the distribution system represented by a spanning tree structure at a specific load condition. Since then, many techniques have been proposed. A branch and bound type 
heuristic algorithm was suggested by Civanlar, Grainger, Yin, and Lee[2], where a simple formula was developed for determination of change in power loss due to a branch exchange. Shirmohammdi and Hong [3] applied optimal power flow analysis to network reconfiguration for loss minimization. More over Baran and $\mathrm{Wu}$ [4] proposed an algorithm to identify branches to be exchanged using heuristic approach to minimize the search for selecting the switching options. In addition Goswami and Basu [5] reported a heuristic algorithm that was based on the concept of optimum flow pattern. The optimum flow pattern with single loop formed by closing a normally open switch was found out, and this flow pattern was established in the radial network by opening a closed switch. This procedure was repeated until the minimum loss configuration was obtained. McDermott, Drezga, and Broadwater [6] proposed a heuristic constructive algorithm that started with all maneuverable switches open, and at each step, the switch that resulted in the minimum increment in the objective function was closed. The objective function was defined as the ratio of incremental losses to incremental load served. Lin, Chin and Yu [7] designed heuristic based switching indices, by utilizing fuzzy notations for the distribution system loss reduction. Taylor and Lubkeman [8] proposed a switch exchange type heuristic method to determine the network configuration for over loads, voltage problem, and for load balancing simultaneously. Its solution scheme set up a decision tree which represented the various operations available, and a best-first search and heuristic rules were used to find feasible switching operations.

The proposed Harmony Search Algorithm (HSA) is used for capacitor placement and solving the network reconfiguration problem to get optimal switching combinations simultaneously in the network to minimize real power losses in the distribution network for dynamic load. The simplicity and effectiveness of the HSA algorithm has led to its application to optimization problems of different areas. HSA algorithm works efficiently in identifying the high performance area of the solution region in a reasonable good time but has average performance at local level search for numerical applications. To improve the performance of the HSA algorithm, various variants of HSA have been proposed. HSA algorithm has various advantages over other metaheuristic algorithms:

- HSA does not need primary value settings of the decision variables.

- For HSA derivative information is unnecessary as it uses stochastic random searches

- In HSA new agent is generated, after considering all of the existing agents.

The method has been tested on standard IEEE 33-bus radial distribution systems for four different season dynamic load levels in a year to demonstrate the performance and effectiveness of the proposed method. The results obtained are encouraging. The remaining part of the paper is organized as follows: Section II gives the problem formulation, Section III discusses the proposed algorithm, Section IV develops the reconfiguration results and discussions and Section V discusses the conclusions.

\section{PROBLEM FORMULATION FOR LOSS MINIMIZATION}

The objective function of the problem is formulated to maximize the power loss reduction in distributed system, which is given by

$$
\begin{aligned}
& \text { Maximize } \mathrm{f}=\max \cdot\left(\Delta P_{\text {Loss }}^{R}+\Delta P_{\text {Loss }}^{D G}\right) \\
& \text { Subjected to } \quad V_{\min } \leq\left|V_{k}\right| \leq V_{\max } \\
& \text { and }\left|I_{k, k+1}\right| \leq \mid I_{k, k+1, \max } \\
& \sum_{k=1}^{n} P_{G k} \leq \sum_{k=1}^{n}\left(P_{k}+P_{\text {Loss }, k}\right)
\end{aligned}
$$


$\operatorname{det}(\mathrm{A})=1$ or $-1($ radial system $)$

$\operatorname{det}(\mathrm{A})=0($ not radial $)$

Power flows in a distribution system are computed by the following set of simplified recursive equations derived from the single-line diagram shown in Fig.1

\subsection{Power Flow Equations}

$$
\begin{aligned}
& P_{k+1}=P_{k}-P_{L o s s, k}-P_{L k+1} \\
& =P_{k}-\frac{R_{k}}{\left|V_{k}\right|^{2}}\left\{P_{k}^{2}+\left(Q_{k}+Y_{k}\left|V_{k}\right|^{2}\right)^{2}\right\}-P_{L k+1} \\
& Q_{k+1}=Q_{k}-Q_{L o s s, k}-Q_{L k+1} \\
& =Q_{k}-\frac{X_{k}}{\left|V_{k}\right|^{2}}\left\{P_{k}^{2}+\left(Q_{k}+Y_{k 1}\left|V_{k}\right|^{2}\right)^{2}\right\}-Y_{k 1}\left|V_{k}\right|^{2}-Y_{k 2}\left|V_{k+1}\right|^{2}-Q_{L k+1} \\
& \left|V_{k+1}\right|^{2}=\left|V_{k}\right|^{2}+\frac{R_{k}^{2}+X_{k}^{2}}{\left|V_{k}\right|^{2}}-2\left(R_{k} P_{k}+X_{k} Q_{k}\right) \\
& =\left|V_{k}\right|^{2}+\frac{R_{k}^{2}+X_{k}^{2}}{\left|V_{k}\right|^{2}}\left(P_{k}^{2}+\left(Q_{k}+Y_{k}\left|V_{k}\right|^{2}\right)^{2}\right) \quad-2\left(R_{k} P_{k}+X_{k}\left(Q_{k}+Y_{k}\left|V_{k}\right|^{2}\right)\right)
\end{aligned}
$$

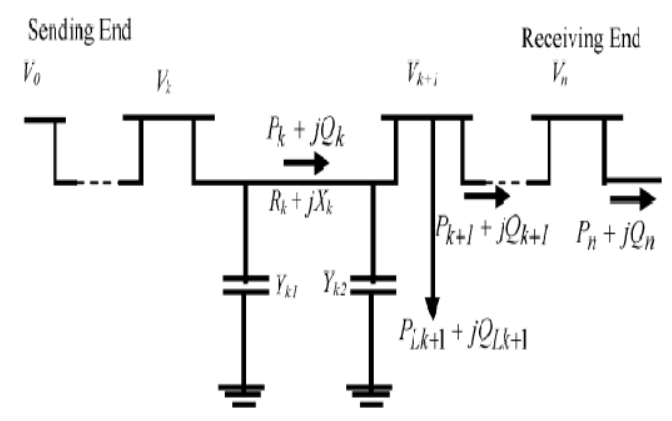

Figure.1 Single-line diagram of a main feeder

The power loss in the line section connecting buses $\mathrm{k}$ and $\mathrm{k}+1$ may be computed as

$$
P_{\text {Loss }}(k, k+)=R_{k} \cdot \frac{\left(P_{k}^{2}+Q_{k}^{2}\right)}{\left|V_{k}\right|^{2}}
$$

The total power loss of the feeder, PT, Loss, may then be determined by summing up the losses of all line sections of the feeder, which is given as

$P_{T, \text { Loss }}=\sum_{k=1}^{n} P_{\text {Loss }}(k, k+1)$ 


\section{PROPOSED METHOD}

The proposed method can be explain in following steps:

Step 1) Obtain the problem and algorithm parameters.

Step 2) Initialize the harmony memory.

Step 3) Improvise a new harmony.

Step 4) Update the harmony memory.

Step 5) Check the termination criterion.

Steps are described as follows [9] \& [10].

\subsection{Obtain the Problem and Algorithm Parameters:}

The optimization problem is generally specified as follows:

Minimize $f(x)$

Subject to $x_{i} \in X_{i}, i=1,2, \ldots \ldots . N$

Where $f(x)$ is an objective function to be solved ; $x$ is the each decision variable sets $x_{i} ; N$ is the number of decision variables; $X_{i}$ is the set for each decision variable with possible range of values, which is $L x_{i} \leq X_{i} \leq U x_{i}$; and $L x_{i}$ and $U x_{i}$ are the lower and upper limit for each decision variable. The HSA parameters are specified in the step as follows. These are the Harmony Memory Size (HMS), or the number of solution agents in the harmony memory; Harmony Memory Considering Rate (HMCR); Pitch Adjusting Rate (PAR); and the Number of Improvisations (NI), or stopping criterion. The harmony memory (HM) is a memory location where all the solution agents (sets of decision variables) are stored. HMCR and PAR are parameters that are used to improve the result agent, which are explained in Step 3.

\subsection{Initialize the Harmony Memory:}

Here, many randomly generated solution agents as HMS are filled in HM matrix

$\mathrm{HM}=\left[\begin{array}{ccccc}x_{1}^{1} & x_{2}^{1} & \cdots & x_{N-1}^{1} & x_{N}^{1} \\ x_{1}^{2} & x_{2}^{2} & \cdots & x_{N-1}^{2} & x_{N}^{2} \\ \vdots & \vdots & \vdots & \vdots & \vdots \\ x_{1}^{H M S-1} & x_{2}^{H M S-1} & \cdots & x_{N-1}^{H M S-1} & x_{N}^{H M S-1} \\ x_{1}^{H M S} & x_{2}^{H M S} & \cdots & x_{N-1}^{H M S} & x_{N}^{H M S}\end{array}\right]$

\subsection{Improvise a New Harmony:}

A New Harmony agent $x^{\prime \rightarrow}=\left(x_{1}^{\prime}, x_{2}^{\prime}, \ldots \ldots . x_{N}^{\prime}\right)$ is produced based on three criteria:

a) Memory consideration,

b) Pitch adjustment, and

c) Random selection.

Producing a new harmony is called improvisation. HMCR, which lies between 0 and 1 and it is the rate of choosing one value from the historical values stored in the HM, while (1-HMCR) is the rate of randomly selecting one value from the possible range of values, as shown in (17):

if $(\operatorname{rand}()<H M C R)$ 


$$
\begin{aligned}
& x_{i}^{\prime} \leftarrow x_{i}^{\prime} \in\left\{x_{i}^{1}, x_{i}^{2}, \ldots \ldots \ldots x_{1}^{H M S}\right\} \\
& \text { else } \\
& x_{i}^{\prime} \leftarrow x_{i}^{\prime} \in X_{i} \\
& \text { end }
\end{aligned}
$$

Where $\operatorname{rand}()$ is a uniformly distributed random number between 0 and 1 and $X_{i}$ is the set of the possible range of values for each decision variable, i.e., $x_{i} \leq X_{i} \leq U x_{i}$. A HMCR of 0.75 reflects that HSA will storically value of HM with $75 \%$ probability. Every component obtained with memory consideration is examined to determine if pitch is to be adjusted. The rate of pitch adjustment as a parameter is given as:

if $(\operatorname{rand}()<P A R)$

$x_{i}^{\prime}=x_{i}^{\prime} \pm \operatorname{rand}(\quad) * b w$

else

$x_{i}^{\prime}=x_{i}^{\prime}$

end

An arbitrary distance bandwidth for the continuous design variable is represented by $b w$. Its value is taken as 1 for problem which is discrete in nature or it can be totally eliminated from the equation for special cases.

\subsection{Update Harmony Memory:}

If the New Harmony agent $x^{\rightarrow \prime}=\left(x_{1}^{\prime}, x^{\prime}{ }_{2}, \ldots \ldots \ldots \ldots x_{N}^{\prime}\right)$ has better fitness function compare to existing or worst harmony in the HM, the new harmony is inserted and worst/existing is excluded.

\subsection{Check Termination Criterion:}

The HSA is completed when the termination criterion has been met. or else, steps 3 and 4 are repeated

\section{TEST RESULT AND DISCUSSION}

The reconfiguration is done on standard IEEE 33 Bus distribution system. The validity and effectiveness of the proposed method is demonstrated. Table.1 [11] represents dynamic load data is for four different seasons in a year. 
Electrical and Electronics Engineering: An International Journal (ELELIJ) Vol 3, No 2, May 2014

\begin{tabular}{|l|l|l|l|l|}
\hline Hour & Winter & Spring & Summer & Fall \\
\hline $12-1$ & 0.4745 & 0.3969 & 0.54 & 0.3717 \\
\hline $1-2$ & 0.4473 & 0.3906 & 0.60 & 0.3658 \\
\hline $2-3$ & 0.4260 & 0.3780 & 0.58 & 0.3540 \\
\hline $3-4$ & 0.4189 & 0.3654 & 0.56 & 0.3422 \\
\hline $4-5$ & 0.4189 & 0.3717 & 0.56 & 0.3481 \\
\hline $5-6$ & 0.5254 & 0.4095 & 0.58 & 0.3835 \\
\hline $6-7$ & 0.6106 & 0.4536 & 0.64 & 0.4248 \\
\hline $7-8$ & 0.6745 & 0.5355 & 0.76 & 0.5015 \\
\hline $8-9$ & 0.6816 & 0.5985 & 0.87 & 0.5805 \\
\hline $9-10$ & 0.6816 & 0.6237 & 0.95 & 0.5841 \\
\hline $10-11$ & 0.6745 & 0.6300 & 0.99 & 0.5900 \\
\hline $11-12 \mathrm{PM}$ & 0.6745 & 0.6237 & 1.00 & 0.5841 \\
\hline $12-1$ & 0.6745 & 0.5859 & 0.99 & 0.5487 \\
\hline $1-2$ & 0.6603 & 0.5796 & 1.00 & 0.5428 \\
\hline $2-3$ & 0.6674 & 0.5670 & 1.00 & 0.5310 \\
\hline $3-4$ & 0.7029 & 0.5544 & 0.97 & 0.5192 \\
\hline $4-5$ & 0.7100 & 0.5670 & 0.96 & 0.5310 \\
\hline $5-6$ & 0.7100 & 0.5796 & 0.96 & 0.5428 \\
\hline $6-7$ & 0.6048 & 0.6048 & 0.93 & 0.5664 \\
\hline $7-8$ & 0.6816 & 0.6174 & 0.92 & 0.5782 \\
\hline $8-9$ & 0.6461 & 0.6048 & 0.92 & 0.5664 \\
\hline $9-10$ & 0.5893 & 0.5670 & 0.93 & 0.5310 \\
\hline $10-11$ & 0.5183 & 0.5040 & 0.87 & 0.4730 \\
\hline $11-12 \mathrm{AM}$ & 0.4473 & 0.4410 & 0.72 & 0.4130 \\
\hline
\end{tabular}

Table.1: Load data for system

Network reconfiguration is the process of changing the distribution network topology by changing the status of switches. Opening and closing of switches can change the amount of power loss which can be seen in Table-2. The standard IEEE 33 Bus distribution system is selected for optimal tie line switching for loss minimization by using methods Genetic Algorithm (GA), Ant colony optimization (ACO) and HSA.

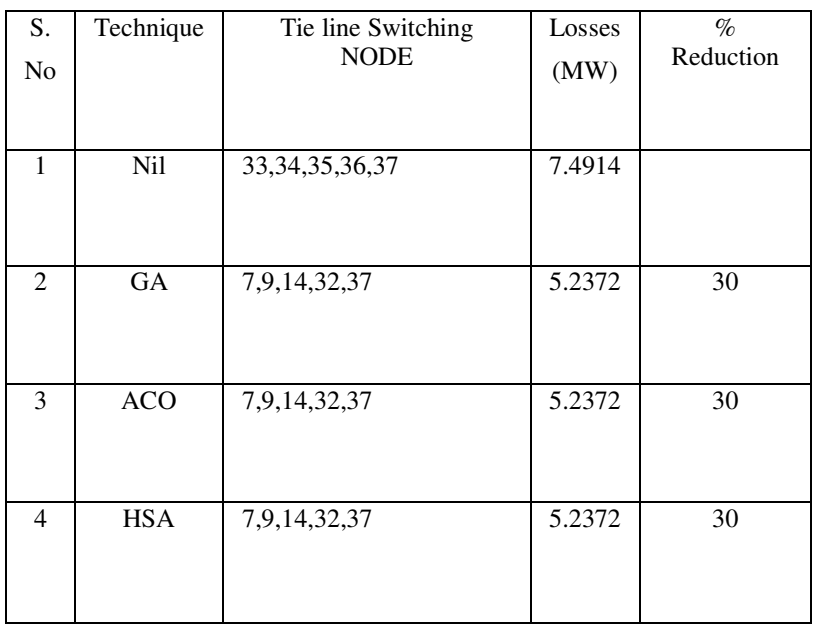

Table.2: Tie Line Switching Result 
The proposed algorithm has been implemented in MATLAB 7.0. Newton Raphson method is employed for calculating the power losses. Normally open switches are 33, 34,35,36,37 creating a power losses of 7.4914 MW. After applying optimal algorithms, open switches selected are $7,9,14,32,37$ and creating a power loss of 5.2373 MW .Thus losses shows $30 \%$ reduction which can also seen from Table-2.

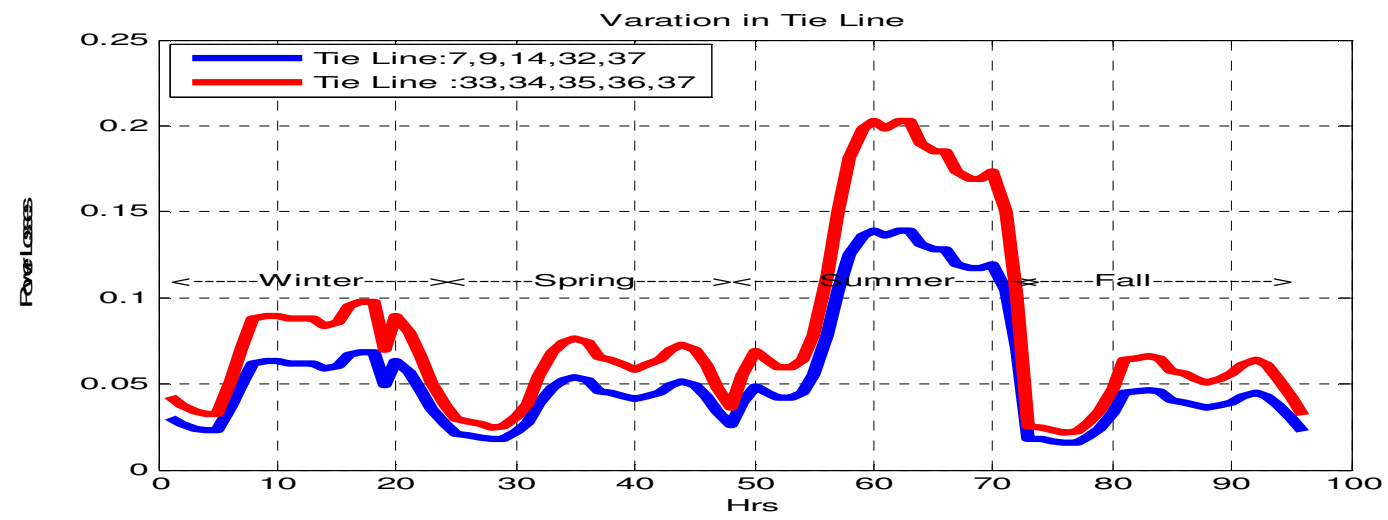

Fig.1: Variation in load

Figure-1 clearly shows variation of power losses for changing load of an year as stated in table-1. Here by selecting optimal tie line switches power losses are minimized for all four seasons and specially in summer when load is at full swing.

Voltage profile before and after reconfiguration is shown in Figure-2. It is observe that the before reconfiguration voltages at bus no 8,13 and 31 was $0.9730,0.964,0.9600$ and 0.9620 pu respectively .

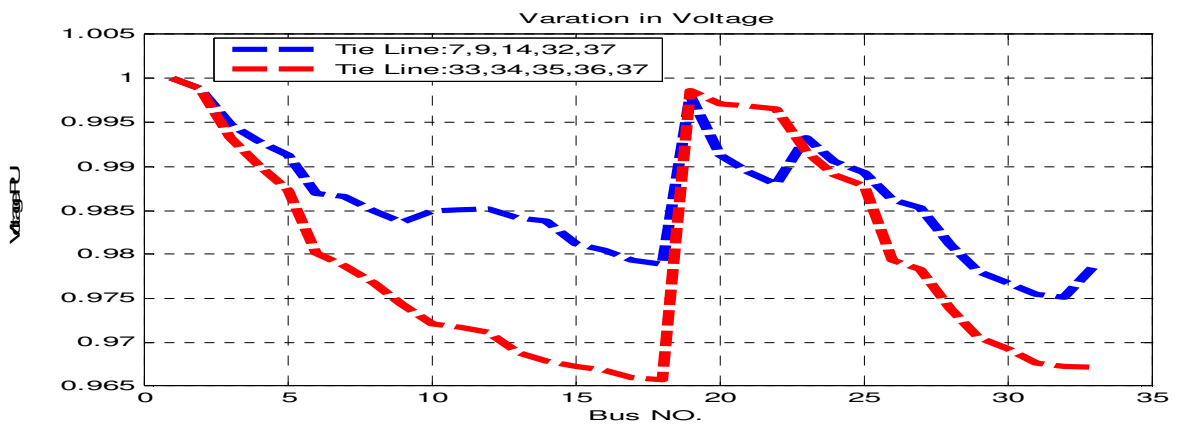

Fig.2: Voltage Profile

After reconfiguration voltages at bus no 8, 13,18 and 31 was $0.9830,0.9820,0.9760$ and 0.9710 pu respectively. This show improves in voltage profile in the network after reconfiguration.

Network reconfiguration and capacitor placement simultaneously using proposed approach is tested on same network. By optimal capacitor placement by proposed approach power losses is reduced from 5.2373 MW to 3.9544 MW which shows $24.70 \%$ reduction as shown in table-3 


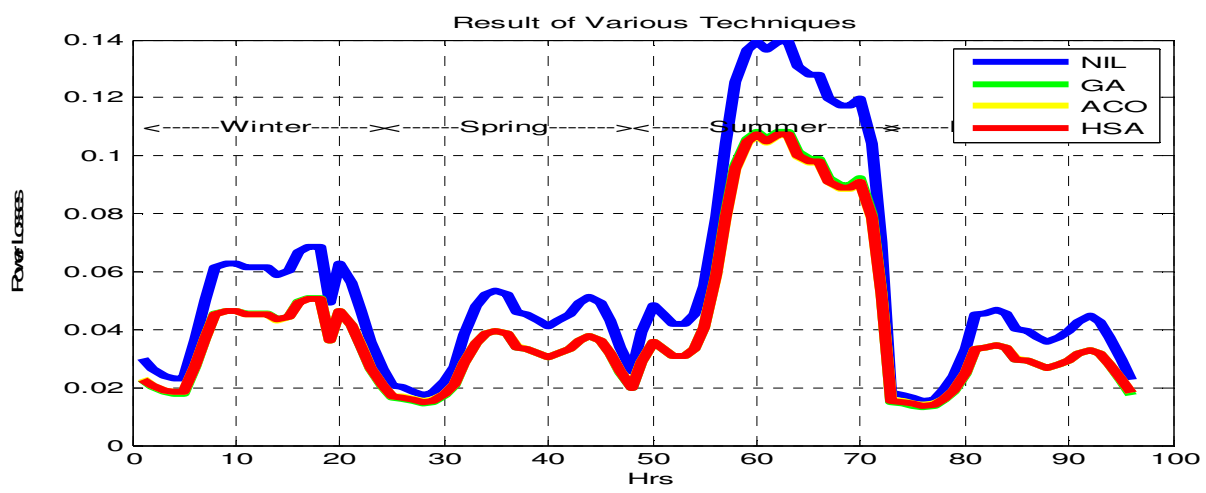

Fig.3: Variation in Load with various Techniques

Figure-3 simply shows the variation of power losses for changing load of in a year as stated in table-1. as similar to figure 1 for tie line switching. Here by selecting optimal capacitor sizes and location, power losses are minimized for all four seasons with variant load.

\begin{tabular}{|l|l|l|l|l|l|l|l|}
\hline S.No. & Technique & $\begin{array}{l}\text { Location } \\
\text { (Bus No.) }\end{array}$ & $\begin{array}{l}\text { Capacitor } \\
\text { Size } \\
\text { (MVAR) }\end{array}$ & $\begin{array}{l}\text { Loss } \\
\text { (MW) }\end{array}$ & $\begin{array}{l}\% \\
\text { Reduction } \\
\text { Of Losses }\end{array}$ & $\begin{array}{l}\text { Time } \\
(\mathrm{Sec})\end{array}$ & $\begin{array}{l}\text { Population, } \\
\text { Iteration }\end{array}$ \\
\hline 01 & NIL & 0 & 0 & 5.2373 & ----- & ----- & ----- \\
\hline 02 & GA & 30 & 0.5905 & 3.9589 & $24.40 \%$ & 9060. & 100,50 \\
\hline 03 & ACO & 30 & 0.6440 & 3.9551 & $24.48 \%$ & 11938 & 100,50 \\
\hline 04 & HSA & 30 & 0.6286 & 3.9544 & $24.70 \%$ & 2069 & 1000,100 \\
\hline
\end{tabular}

Table.3: Optimum Result

Computational result also shows that the optimum result as shown in Table-3 is available by GA and ACO attempt methods simultaneously with capacitor placement at 50 iteration with population size of 100 . The average CPU time taken by the process to carry out the simulation is $9060 \mathrm{sec}$ and $11938 \mathrm{sec}$ respectively. While optimum solution is available by proposed method is available at 100 iteration but of population size of 1000 and average CPU time taken by the process to carry out the simulation is $2069 \mathrm{sec}$. which is comparatively much less than previous attempt methods.

Fig-4 shows variation of voltages with bus number before and after placement of optimal capacitors using different optimal techniques. Nearly similar to Figure 2 for tie line switching. Here voltage profile is seen clearly improve specially from bus no 25 to 33 voltage profile of all optimal technique stated in paper provide nearly similar result in improving the voltage profile 


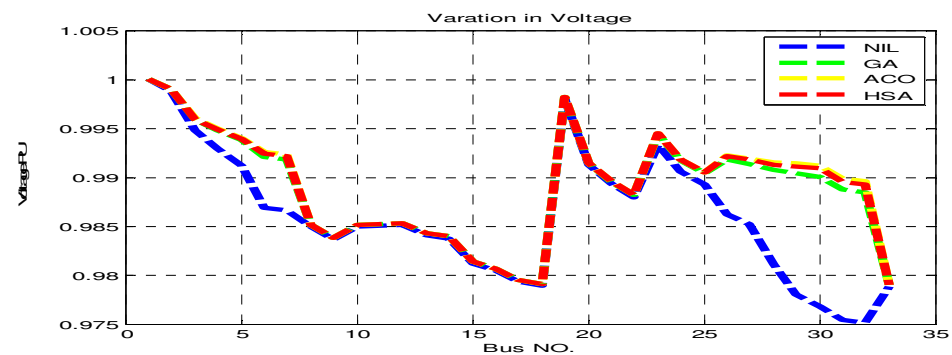

Fig. 4: Variation in voltage with capacitor placement

HSA has shown that its performance is much more satisfactory than several other metaheuristic algorithm to solve network reconfiguration and capacitor placement.

\section{CONCLUSION}

In this paper proposed "HSA" has been tested on Standard IEEE 33 bus distribution system. Optimal network reconfiguration and capacitor placement problem are solved for the distribution system to achieve the minimum power loss in the system at reasonable amount of time. In the optimization process the applied constraints is voltage of buses. Minimum losses with improved voltage profile are achieved. Optimal capacitor placement can control the flow of reactive power in distribution system which turns lead to power loss reduction. It is conclude that simultaneously solving of this problem will lead to better result than other approaches. The power distribution utilities can prioritize their network improvement optimally using the above result.

\section{REFERENCES}

[1] A. Merlin, H. Back, "Search for a minimal-loss operating spanning tree configuration in an urban power distribution system" Proceedings of 5th Power System Computation Conference (PSCC), Cambridge, UK, 1975, pp. 1-18.

[2] S. Civanlar, J. J. Grainger, H. Yin, and S. S. H. Lee, "Distribution feeder reconfiguration for loss reduction,” IEEE Trans. Power Del., vol. 3, no. 3, pp. 1217-1223, Jul. 1988.

[3] D. Shirmohammadi and H. W. Hong, "Reconfiguration of electric distribution networks for resistive line loss reduction," IEEE Trans. Power Del., vol. 4, no. 2, pp. 1492-1498, Apr. 1989.

[4] M. E. Baran and F. F. Wu, "Network reconfiguration in distribution systems for loss reduction and load balancing," IEEE Trans. Power Del., vol. 4, no. 2, pp. 1401-1407, Apr. 1989.

[5] S. K. Goswami and S. K. Basu, "A new algorithm for the reconfiguration of distribution feeders for loss minimization,” IEEE Trans. Power Del., vol. 7, no. 3, pp. 1484-1491, Jul. 1992.

[6] T. E. McDermott, I. Drezga, and R. P. Broadwater, "A heuristic nonlinear constructive method for distribution system reconfiguration," IEEE Trans. Power Syst., vol. 14, no. 2, pp. 478-483, May 1999.

[7] W. M. Lin, H. C. Chin, G. J. Yu, "An effective algorithm for distribution feeder loss by switching operations", IEEE Trans. on Power delivery, pp 597 - 602, 1999.

[8] T. Taylor, D. Lubkeman, "Implementation of heuristic search strategies for distribution feeder reconfiguration”, IEEE Trans. on Power delivery, vol. 5, No. 1, pp 239 - 246, January 1990.

[9] R. Srinivasa Rao, S. V. L. Narasimham, "A New Heuristic Approach for Optimal Network Reconfiguration in Distribution Systems", International Journal of Applied Science, Engineering and Technology, vol. 5, No. 1, 2009.

[10] R. Srinivasa Rao, K. Ravindra,K.Satish , S. V. L. Narasimham "Power Loss Minimization in Distribution System using Network Reconfiguration in Presence of Distributed Generation", IEEE Trans. on Power system, vol. 28, No. 1, pp 317 - 325, Feburary 2013. 
Electrical and Electronics Engineering: An International Journal (ELELIJ) Vol 3, No 2, May 2014

[11] Y. M. Atwa, E. F. El Saadany, M. M. A. Salama, and R. Seethapathy "Optimal Renewable Resources Mix for Distribution System Energy Loss Minimization”, IEEE Trans. on Power Systems, vol. 25. no.1, pp. 360-370, Feb. 2010.

\section{Authors}

Pushpendra Singh, has obtain degree in Bachelor of Engg. In Electrical from Rajasthan University, Jaipur in 2005.He completed his M.Tech In power system From MNIT Jaipur in 2011.His area of research is Distribution system. Presently working as Asst. Professor in GWEC,Ajmer,India

Komal Arora, has obtain degree in Bachelor of Engg. In Electrical from Rajasthan University, Jaipur in 2004. She completed her M.tech in Power System from RTU Kota in 2009. Her area of research in Optimal Power Flow. Presently working as Asst. Professor in GWEC,Ajmer, India
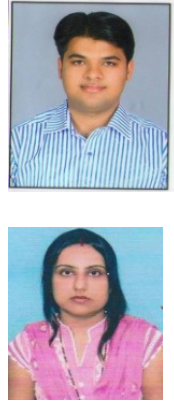